\title{
Efficacy of Tegoprazan for Improving the Susceptibility of Antimicrobial Agents against Antibiotic-Resistant Helicobacter pylori
}

\author{
Jung Won Lee ${ }^{1,2}$, Nayoung Kim ${ }^{1,3}$, Ryoung Hee Nam¹, Jeong Eun Yư ${ }^{1}$, Joo Hee Son'1, Sun Min Lee ${ }^{1}$, and Dong Ho Lee ${ }^{1,3}$ \\ ${ }^{1}$ Department of Internal Medicine, Seoul National University Bundang Hospital, Seongnam, ${ }^{2}$ Department of Internal Medicine, \\ Samsung Changwon Hospital, Sungkyunkwan University School of Medicine, Changwon, and ${ }^{3}$ Department of Internal Medicine and \\ Liver Research Institute, Seoul National University College of Medicine, Seoul, Korea
}

\section{Article Info}

Received August 1, 2020

Revised October 4, 2020

Accepted October 5, 2020

Published online November 17, 2020

\section{Corresponding Author}

\section{Nayoung Kim}

ORCID https://orcid.org/0000-0002-9397-0406

E-mail nayoungkim49@empas.com, nakim49@snu.ac.kr

\begin{abstract}
Background/Aims: Favorable outcomes of potassium-competitive acid blocker (PCAB)-containing eradication therapy have been reported. In fact, tegoprazan, a recently developed PCAB, was presumed to show good eradication efficacy even for resistant Helicobacter pylori. We aimed to investigate the anti- $H$. pylori efficacy of tegoprazan compared with that of vonoprazan.

Methods: A total of 220 resistant clinical $H$. pylori isolates were utilized. The anti- $H$. pylori efficacy of PCABs was determined by evaluating the minimum inhibitory concentrations (MICs) of clarithromycin, fluoroquinolone, metronidazole, and amoxicillin in combination with vonoprazan or tegoprazan by the agar dilution method. The impact of the mutations responsible for resistance development, such as 23S rRNA, gyrA, $r d x A$, frxA, and pbp1 mutations, was also analyzed.

Results: $H$. pylori growth was significantly inhibited in a medium containing $1 \mu \mathrm{g} / \mathrm{mL}$ clarithromycin with tegoprazan $(128 \mu \mathrm{g} / \mathrm{mL})$. The MICs of clarithromycin $(46.3 \%)$, fluoroquinolone $(46.7 \%)$, metronidazole (55.6\%), and amoxicillin (34.5\%) against resistant $H$. pylori isolates improved after tegoprazan administration. Tegoprazan demonstrated more frequent susceptibility acquisition with metronidazole than with vonoprazan $(20.6 \%$ vs $4.7 \%, p=0.014)$. However, there were no significant differences depending on the mutational status of each antimicrobial agent.

Conclusions: Tegoprazan administration may improve the susceptibility of antimicrobial-resistant H. pylori, independent of acid suppression. (Gut Liver 2021;15:53-60)
\end{abstract}

Key Words: Helicobacter pylori; Potassium-competitive acid blocker; Eradication; Resistance

\section{INTRODUCTION}

Potassium-competitive acid blocker (PCAB), a recently developed drug, strongly inhibits gastric acid secretion via different mechanisms from proton pump inhibitor (PPI). Compared to conventional PPI-containing eradication therapies, the first developed PCAB, vonoprazan, demonstrated rapid and potent acid suppression and more effective eradication results. ${ }^{1}$ The superior efficacy of vonoprazan-containing eradication treatment has been demonstrated in several studies and is mainly explained by its rapid and potent suppression of gastric acid secretion. ${ }^{2}$ However, favorable outcomes of vonoprazan-containing Helicobacter pylori eradication treatment, even the clarithromycin-resistant strains that could not be overcome with conventional PPI-containing treatments, ${ }^{3}$ enabled the proposal of a hypothesis for the existence of another mechanism besides acid suppression.

Following the success of vonoprazan, different PCAB medicines such as tegoprazan have been developed. ${ }^{4,5}$ Tegoprazan demonstrated highly selective potassiumcompetitive acid blocking efficacy and is currently proven to be effective for managing gastroesophageal reflux disease. ${ }^{5,6}$ Therefore, the rapid and potent anti-acid efficacy of tegoprazan is considered optimal for $H$. pylori eradication therapy. However, there are limited data on tegoprazan for $H$. pylori eradication efficacy. Therefore, we hypothesized that PCAB, including tegoprazan, could improve antimicrobial susceptibility to antibiotics in vitro, especially in the presence of important mutations that inhibit the suc- 
cess of $H$. pylori eradication in vivo. Thus, the aim of this study was to evaluate the decrease in minimum inhibitory concentration (MIC) by tegoprazan compared with vonoprazan in the resistant $H$. pylori isolates obtained from patients with different upper gastrointestinal tract diseases. In addition, we sought to determine whether the improved susceptibility could differ according to the mutations responsible for antimicrobial resistance.

\section{MATERIALS AND METHODS}

\section{Study material and subject}

Between December 2003 and December 2019, patients with $H$. pylori infection were consecutively enrolled at the Seoul National University Bundang Hospital located in Gyeonggi province, near Seoul, Korea. Patients' demographic and clinical data, including age, sex, H. pylori eradication results, and endoscopic findings, were collected. $H$. pylori infection was defined by a positive rapid urease test (Campylobacter-like organism test; Delta West, Bentley, Australia), histological evidence of $H$. pylori by modified Giemsa staining of the gastric mucosal biopsy samples obtained from the lesser curvature and greater curvature of the mid-antrum and mid-body, or a positive ${ }^{13} \mathrm{C}$-urea breath test. All subjects provided informed consent, and the study protocol was approved by the Ethics Committee of the Seoul National University Bundang Hospital (IRB number: B-1901-514-305). Tegoprazan was provided by HK inno.N Corporation (Seoul, South Korea). Vonoprazan (TAK-438) was purchased from Bide Pharmatech Ltd (Shanghai, China).

\section{Efficacy against resistant $\boldsymbol{H}$. pylori isolates}

We experimented to prove the anti- $H$. pylori efficacy of PCAB agents. First, clinical $H$. pylori isolates susceptible to all antimicrobial agents used for $H$. pylori eradication were selected. Different concentrations of PCAB agents were examined against these isolates. Vonoprazan and tegoprazan were mixed with dimethyl sulfoxide with $\mathrm{HCl}$ and administered at an initial concentration of $1 \mu \mathrm{g} / \mathrm{mL}$ with gradual increase up to $128 \mu \mathrm{g} / \mathrm{mL}$. If no efficacy was observed with PCABs alone, additional experiments were performed using a clarithromycin-containing medium. Through this experiment, the concentration of PCABs that could improve susceptibility to antimicrobial agents was recorded, and this concentration was used in the next experiment.

To determine whether PCABs are effective against resistant $H$. pylori isolates, a series of experiments was conducted on clinical $H$. pylori isolates resistant to each of the four antimicrobial agents, clarithromycin, metronidazole, fluoroquinolone, and amoxicillin, which have a decisive impact on successful eradication treatment. MIC changes before and after PCAB administration were determined; in addition, we determined whether there are changes beyond the breakpoint of resistance.

\section{Culture and antimicrobial susceptibility test}

For $H$. pylori isolation, mucosal biopsy samples were obtained from the lesser and greater curvatures in the mid-antrum and body of the stomach; these samples were then cultured for $H$. pylori isolation. The MICs against the isolated bacterium to antibiotics (Sigma Chemical Co., St. Louis, MO, USA) were examined by agar dilution. Susceptibility testing reference was based on the recommendations of the Clinical and Laboratory Standards Institute. The bacteria were sub-cultured on Mueller-Hinton agar supplemented with 5\% defibrinated sheep blood for 48 hours. The bacterial suspension, adjusted to $1 \times 10^{7}$ colonyforming units, was inoculated directly onto an antibioticcontaining agar dilution plate. MICs were determined after 72 hours of incubation. The range of concentrations used to evaluate resistance to amoxicillin, levofloxacin, and moxifloxacin was $<0.125$ to $>8 \mu \mathrm{g} / \mathrm{mL}$. For clarithromycin, the range was $<0.25$ to $>4 \mu \mathrm{g} / \mathrm{mL}$, whereas for metronidazole, it was $<2$ to $>64 \mu \mathrm{g} / \mathrm{mL}$. The resistance breakpoints for amoxicillin and metronidazole were $\geq 0.5$ and $>8 \mu \mathrm{g} /$ $\mathrm{mL}$, respectively. The breakpoint for amoxicillin and metronidazole resistance was determined according to that specified by the European Committee on Antimicrobial Susceptibility Testing guidelines. The breakpoint for clarithromycin was $>1.0 \mu \mathrm{g} / \mathrm{mL}$ according to Clinical and Laboratory Standards Institute guidelines, whereas that for levofloxacin and moxifloxacin was provisionally defined as $\geq 1.0 \mu \mathrm{g} / \mathrm{mL}^{7.8}$

\section{Determination of mutations affecting the antibiotic resistance of $\boldsymbol{H}$. pylori}

Mutations in 23S rRNA, $g y r A, r d x A$, frx $A$, and $p b p 1$ are decisive genetic mutations responsible for resistant $H$. pylori. ${ }^{8-11}$ A series of experiments was performed to confirm the above mutations using ATCC 43504 wild-type $H$. pylori as a control. Briefly, $H$. pylori genomic DNA was extracted from the isolates. Specimens were homogenized in proteinase $\mathrm{K}$ solution $(20 \mathrm{mmol} / \mathrm{L}$ Tris- $\mathrm{HCl}[\mathrm{pH} 8.0]$, $10 \mathrm{mmol} / \mathrm{L}$ ethylenediaminetetraacetic acid, $0.5 \%$ sodium dodecyl sulfate, and $10 \mathrm{mg} / \mathrm{mL}$ proteinase $\mathrm{K}$ ) using a sterile micropestle and were incubated for 3 hours. DNA was isolated by phenol/chloroform extraction and ethanol precipitation. Polymerase chain reaction amplification for $23 \mathrm{~S}$ rRNA, $g y r A, r d x A$, frx $A$, and $p b p 1$ was performed using the primers specified in Table $1 .^{8-11}$ Amplification was 
conducted using a thermal cycler (MJ PTC-0200; Bio-Rad Laboratories, Waltham, MA, USA), ${ }^{8,12}$ Polymerase chain reaction amplification comprised 35 cycles of 1-minute denaturation at $94^{\circ} \mathrm{C}, 1$-minute annealing at $57^{\circ} \mathrm{C}$, and 1 -minute extension at $72^{\circ} \mathrm{C}$. The amplified product was then purified. Sequencing was performed for the two strands of the non-restricted amplicons using the ABI PRISM 377XL DNA sequencer (Applied Biosystems, Foster City, CA, USA).

\section{Statistical analyses}

We assumed the difference is estimated to be about $10 \%$ between tegoprazan and vonoprazan regarding the result of the study of eosinophilic esophagitis. Based on this result, it was estimated that 50 strains per group would be required assuming the effect size of $0.5, \alpha$-error 0.05 , and $1-\beta 0.80$. The chi-square test or Fisher exact test was used to analyze categorical variables. Continuous variables were reported as means \pm standard deviations and were compared using the Student t-test. A logistic regression analysis was conducted to identify the independent risk factor of improved antimicrobial susceptibility with $\mathrm{PCAB}$ administration. Multiple factors were included in the logistic regression, including age, sex, clinical disease, type of PCABs, and the mutations responsible for resistance development. All statistical tests were two-tailed, and p-value $\leq 0.05$ were considered to indicate statistical significance. All statistical analyses were performed using the SPSS version 19.0 software (IBM Corp., Armonk, NY, USA).

\section{RESULTS}

\section{Anti-H. pylori efficacy of PCABs}

$H$. pylori growth was not significantly inhibited after the administration of vonoprazan or tegoprazan alone. Therefore, subsequent experiments using clarithromycincontaining media were performed. Significant and definite inhibition of $H$. pylori growth was confirmed using clarithromycin $1 \mu \mathrm{g} / \mathrm{mL}$ with tegoprazan $128 \mu \mathrm{g} / \mathrm{mL}$ or vonoprazan $128 \mu \mathrm{g} / \mathrm{mL}$. Based on these results, vonoprazan and tegoprazan $128 \mu \mathrm{g} / \mathrm{mL}$ were determined to be adequate concentrations for confirming the anti- $H$. pylori efficacy of PCABs.

\section{Characteristics of $\boldsymbol{H}$. pylori isolates}

Resistance isolates of clarithromycin, fluoroquinolone, metronidazole, and amoxicillin were 54, 45, 63, and 58, respectively. The number of strains of each group differed depending on the success rate during $H$. pylori culture and MIC test experiment. There were no statistical differences between the groups for age, sex, and clinical diagnosis. The mutations known to have a decisive role in the development of resistance to each antimicrobial agent assessed in this study were also investigated. In particular, A2143, gyrA N87, $r d x A$, and pbp1 (D479 or T593) mutations were classified as driver mutations as they were reported to be closely related to eradication treatment failure. ${ }^{8-11}$ Clarithromycin, fluoroquinolone, metronidazole, and amoxicillin-resistant strains had driver mutations at 55.6\%, $44.4 \%, 54.0 \%$, and $86.2 \%$. The isolates with genetic mutations but lacking driver mutations were classified to have passenger mutations. All $H$. pylori eradication treatments performed in this study were the same protocol as the previous study. ${ }^{13}$

\section{MIC changes after PCAB administration}

Regardless of the degree, increased MIC value of antimicrobial agents against the strains was designated as "MIC improvement." If the MIC value improved and exceeded the resistance breaking point of the antimicrobial agent, its result was termed as "susceptibility acquisition." The improvement in susceptibility acquired by PCAB administration is presented in Fig. 1. Clarithromycin (46.3\%), fluoroquinolone (46.7\%), metronidazole (55.6\%), and amoxicillin (34.5\%) showed more than one level of MIC improvement by tegoprazan administration. Instances of MIC values exceeding three levels or breakpoints of resistance were identified. Tegoprazan demonstrated the highest overall improvement rate for the MIC value (55.6\%) and metronidazole susceptibility (20.6\%). In contrast, clarithromycin (42.6\%), fluoroquinolone (33.3\%), metro-

Table 1. Primer Sets Used for Real Time Polymerase Chain Reaction

\begin{tabular}{|c|c|c|}
\hline Target & Forward & Reverse \\
\hline 23S rRNA & CGTAACTATAACGGTCCTAAG & TTAGCT AACAGA AACATC AAG \\
\hline gyrA & TTTAGCTTATTCAATGAGCGT & GCAGACGGCTTGGTAGAATA \\
\hline$r d x A$ & ATGGTAATTGTTTCGTTAGGG & СTCCTTGAACTTTAATTTAG \\
\hline frxA (first) & TGGATATGGCAGCCGTTTA & GGTTATCAAAAAGCTAACAGCG \\
\hline frxA (second) & GCGAATTGGATATGGCAGCC & ACGCCAAGCTTTTTATGGTTG \\
\hline pbp1 (first) & GCTTTGCTATCTCACCCATT & TCAATTACGGAGATGTTAAAGTC \\
\hline pbp1 (second) & CTTTCAATGAGCATGGGTTT & CGTAGATGAAGTGTTGAAGCAA \\
\hline
\end{tabular}



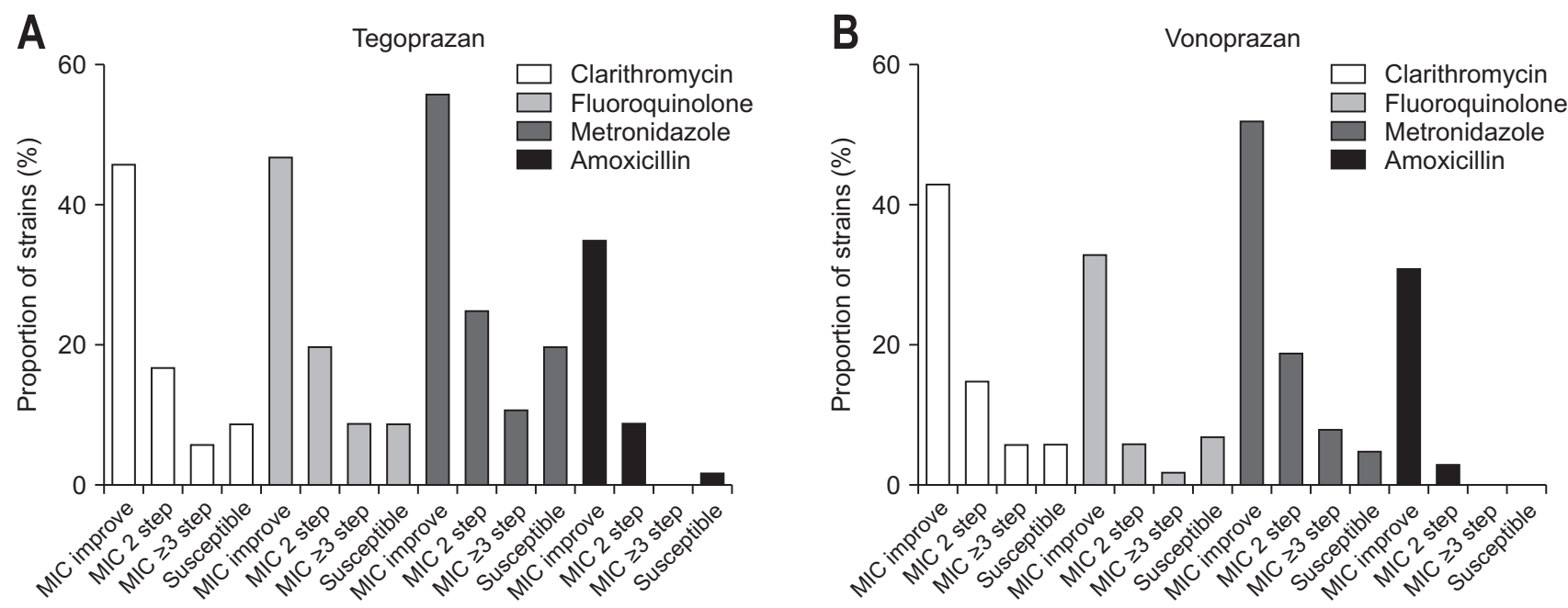

Fig. 1. Effect of tegoprazan and vonoprazan on the antibiotic susceptibility of Helicobacter pylori. (A) Effect of improving susceptibility acquired by the addition of tegoprazan. Clarithromycin (46.3\%), fluoroquinolone (46.7\%), metronidazole (55.6\%), and amoxicillin (34.5\%) increased the minimum inhibitory concentration (MIC) by more than 1-fold relative to that of tegoprazan. The highest overall improvement rate in the MIC was $55.6 \%$, and the acquired susceptibility rate of metronidazole was $20.6 \%$. (B) Effect of improving susceptibility acquired by the addition of vonoprazan. Clarithromycin (42.6\%), fluoroquinolone (33.3\%), metronidazole (52.4\%), and amoxicillin (31.0\%) improved the MIC. Clarithromycin (5.6\%), fluoroquinolone $(6.7 \%)$, and metronidazole $(4.7 \%)$ acquired susceptibility.

nidazole (52.4\%), and amoxicillin (31.0\%) showed more than one level of MIC improvement following vonoprazan administration (Fig. 1B). Clarithromycin (5.6\%), fluoroquinolone (6.7\%), and metronidazole (4.7\%) were found to have susceptibility acquisition by vonoprazan administration. Changes in MIC of antimicrobial agents according to PCAB administration for each strain are presented in Fig. 2.

\section{Comparison of MICs and susceptibilities between PCABs}

We compared MIC improvement and susceptibility acquisition between vonoprazan and tegoprazan. Tegoprazan demonstrated more frequent MIC improvement and susceptibility acquisition than vonoprazan for all antimicrobial agents in the present study (Fig. 3). However, statistical significance was confirmed only in the susceptibility acquisition with metronidazole ( $\mathrm{p}=0.014$ ) (Fig. 3B).

\section{Factor-associated susceptibility changes after PCAB administration for each antibiotic agent for important mutations}

For each antimicrobial agent, a series of statistical analyses was performed to identify the independent risk factors that enabled MIC improvement and susceptibility acquisition. There were no statistically significant differences between the groups in the mutational status of each antimicrobial agent (Table 2). For logistic regression analysis, the independent risk factors significantly related to MIC improvement and susceptibility acquisition were not identified.

\section{DISCUSSION}

In the present study, H. pylori isolates with clarithromycin (46.3\%), fluoroquinolone (46.7\%), metronidazole (55.6\%), and amoxicillin (34.5\%) resistance were found to demonstrate MIC improvement following tegoprazan administration. In addition, tegoprazan demonstrated more frequent susceptibility acquisition with metronidazole than vonoprazan ( $20.6 \%$ vs $4.7 \%$, $\mathrm{p}=0.014)$. Such findings suggest that tegoprazan administration could improve the susceptibility of antimicrobial-resistant $H$. pylori independent of acid suppression.

PPI has been an essential element in $H$. pylori eradication therapy. ${ }^{14,15}$ In addition, $H$. pylori density might be lower and false-negative diagnosis could occur in patients administered PPIs. ${ }^{16}$ The use of high-dose PPI twice daily increases the efficacy of eradication therapy. ${ }^{14,17}$ Thus, it is generally accepted that PPIs exert anti- $H$. pylori activity. ${ }^{14,18}$ Therefore, guidelines for the management of $H$. pylori infection mainly recommend PPI twice per day. Unfortunately, PPI requires 3 to 5 days to reach its maximum anti-acid effect. During this period, the acid suppression required for $H$. pylori eradication could be inadequate.

PCABs emerged as the mainstream treatment for gastroesophageal reflux disease following PPI. PCABs inhibit acid secretion in gastric parietal cells. Vonoprazan has been clinically available since 2015 in Japan and has demonstrated more rapid and potent acid suppression relative to PPIs. Vonoprazan is regarded as an optimal alternative to PPIs for $H$. pylori eradication therapy and is more commonly 


\section{A Clarithromycin}

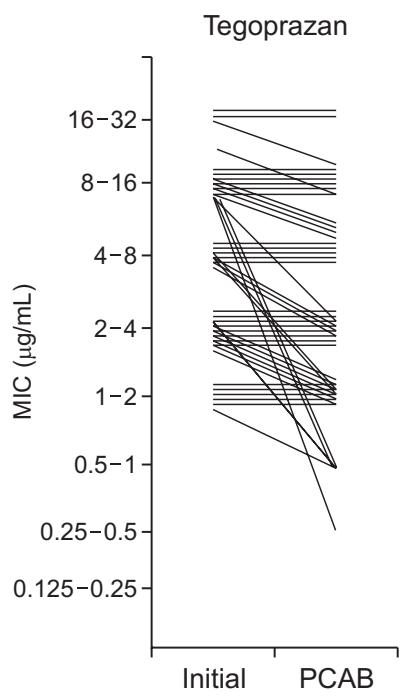

C Metronidazole

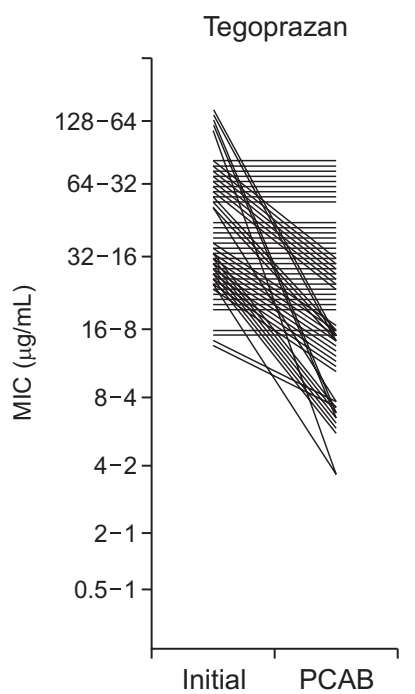

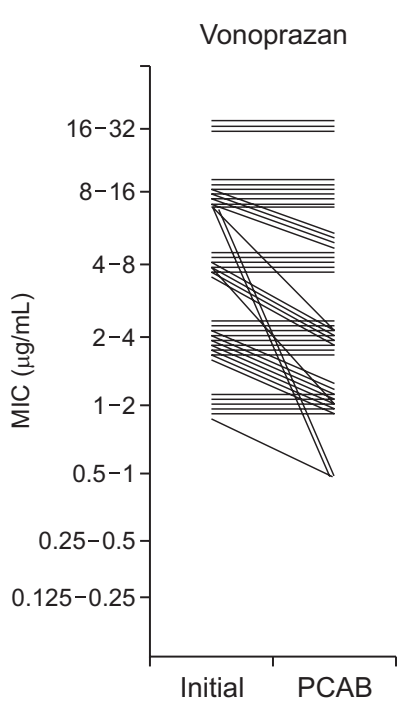

B Fluoroquinolone
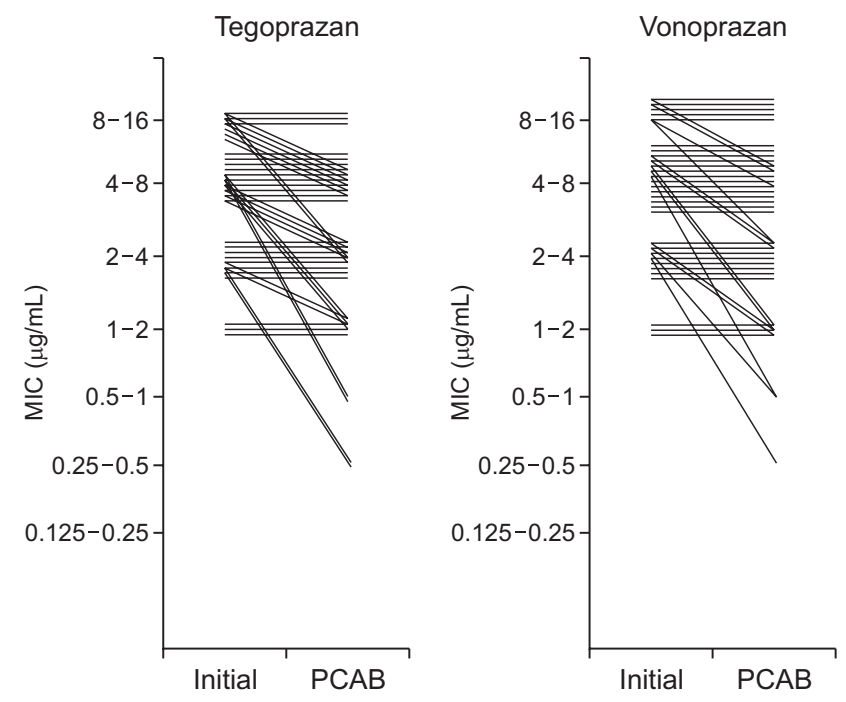

D Amoxicillin
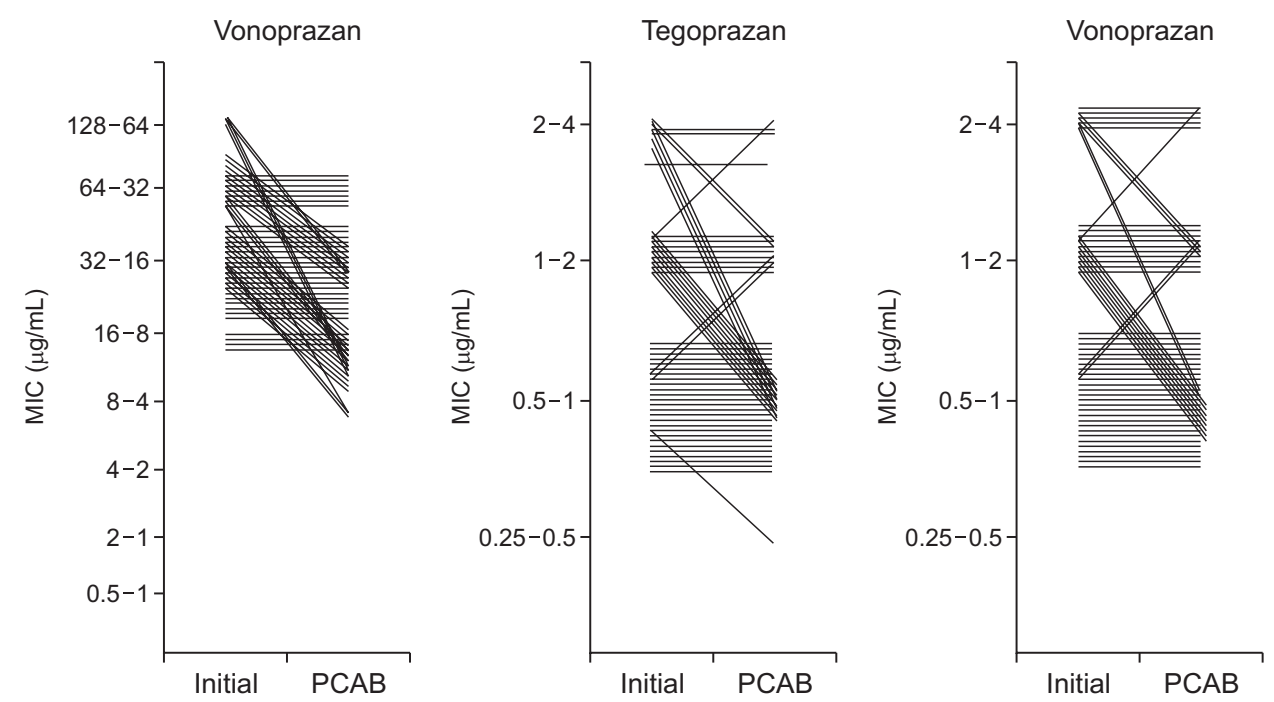

Fig. 2. Changes in the minimum inhibitory concentration (MIC) according to potassium-competitive acid blocker (PCAB) administration in each strain. Results of the addition of clarithromycin (A), fluoroquinolone (B), metronidazole (C), and amoxicillin (D) relative to those of tegoprazan or vonoprazan are presented.

used in eradication therapy in Japan instead of PPI. ${ }^{1}$ In addition, favorable outcomes of PCAB-containing eradication therapy have been reported. The relatively high eradication success rate of Japan is postulated to result from the use of PCAB-containing eradication therapy despite the increasing antimicrobial resistance rate. ${ }^{1}$ However, in the clarithromycin-resistant $H$. pylori strain, vonoprazan-containing eradication therapy demonstrated a higher eradication success rate compared to PPI $(82.0 \%$ vs $40.0 \%){ }^{3}$ Moreover, the superior efficacy of vonoprazan-containing eradication therapy is not limited to vonoprazan therapy. In the third-line salvage treatment, vonoprazan-containing high-dose-dual therapy recently reported favorable outcomes. These favorable outcomes of the PCAB-containing eradication therapy supports the hypothesis regarding the presence of anti- $H$. pylori mechanism of PCABs, besides anti-acidic efficacy. ${ }^{3}$ Tegoprazan was recently approved for use in $H$. pylori eradication treatment of patients with peptic ulcer and/or chronic atrophic gastritis in Korea. ${ }^{5}$ Therefore, based on the knowledge of resistance, the present investigators aim to identify the effect of PCABs on $H$. pylori strains with antimicrobial resistance and resistancerelated genetic mutation by tegoprazan.

In the present study, a significant number of antimicro- 

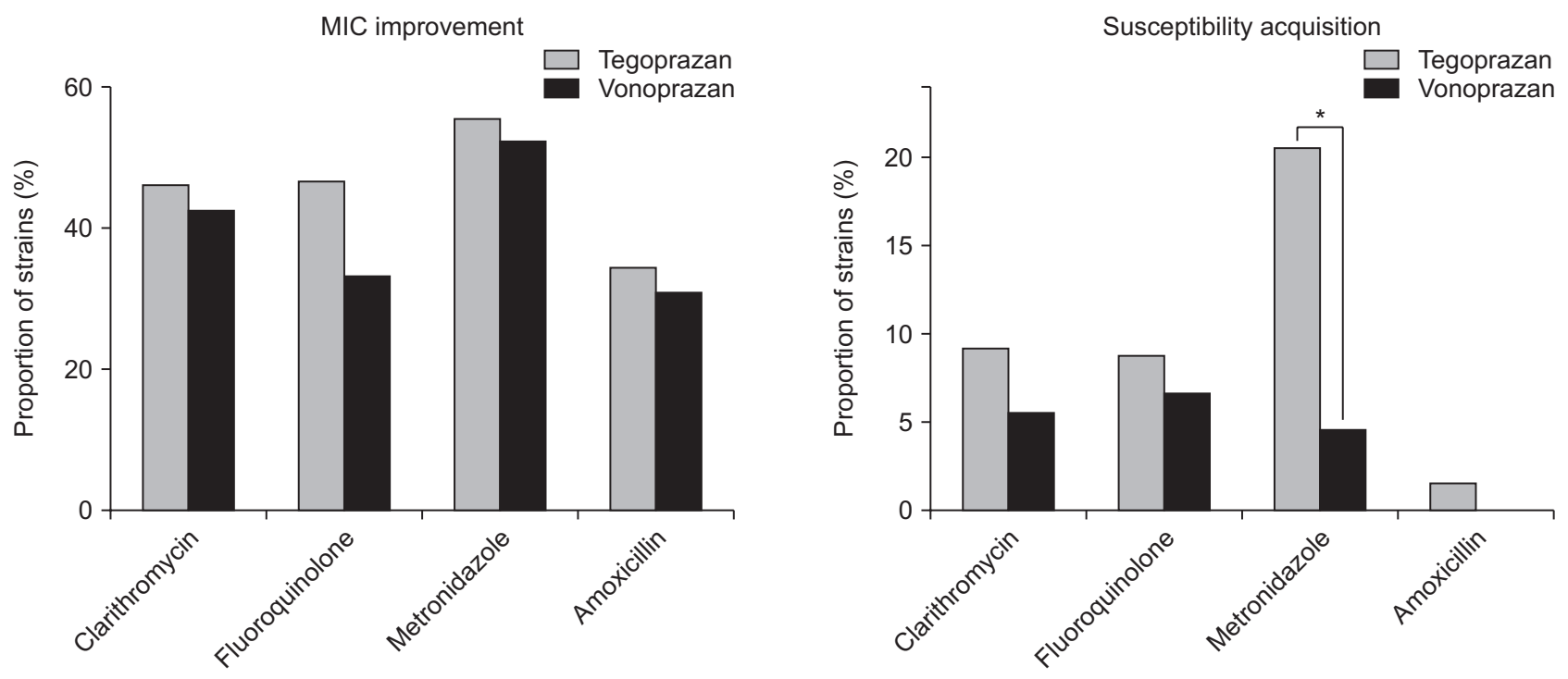

Fig. 3. Comparison of the improved susceptibility of Helicobacter pylori to vonoprazan and tegoprazan. (A) There was no difference in the minimum inhibitory concentration (MIC) between vonoprazan and tegoprazan. (B) Tegoprazan demonstrated more frequent susceptibility acquisition with metronidazole than with vonoprazan $(20.6 \%$ vs $4.7 \%, p=0.014)$. *Statistically significant, $p<0.05$.

Table 2. Effect of Improving the Susceptibility According to Mutations Reflecting Resistance at Tegoprazan

\begin{tabular}{|c|c|c|c|c|}
\hline \multicolumn{3}{|c|}{ Antimicrobial agent } & \multirow{2}{*}{$\frac{\text { MIC improvement, No. (\%) }}{15 / 30(50.0)}$} & \multirow{2}{*}{$\frac{\text { Susceptibility acquired, No. (\%) }}{4 / 30(13.3)}$} \\
\hline Clarithromycin (23S rRNA) & Driver & A2143 & & \\
\hline & No mutation & No mutation & $10 / 23(43.3)$ & $1 / 23(4.3)$ \\
\hline & $\mathrm{p}$-value & & 0.883 & 0.432 \\
\hline \multirow[t]{4}{*}{ Fluoroquinolone (gyrA) } & Driver & N87 & $10 / 20(50.0)$ & $2 / 20(10.0)$ \\
\hline & Passenger & D91 only & $10 / 20(50.0)$ & $2 / 20(10.0)$ \\
\hline & No mutation & No mutation & $1 / 5(20.0)$ & \\
\hline & p-value & & 0.525 & 1.000 \\
\hline \multirow[t]{3}{*}{ Metronidazole $(r d x A)$} & Driver & $r d x A$ or $f r x A$ & $26 / 49(53.1)$ & $9 / 49(18.4)$ \\
\hline & No mutation & No mutation & $9 / 14(64.3)$ & $4 / 14(28.6)$ \\
\hline & p-value & & 0.550 & 0.461 \\
\hline \multirow[t]{5}{*}{ Amoxicillin (pbp1) } & Driver & D479 \& T593 & $8 / 25(32.0)$ & \\
\hline & Driver & D479 only & $4 / 11(36.4)$ & $1 / 11(9.1)$ \\
\hline & Driver & T593 only & $6 / 14(42.9)$ & \\
\hline & Passenger & No 479,593 & $2 / 8(25.0)$ & \\
\hline & $p$-value & & 0.901 & 0.328 \\
\hline
\end{tabular}

MIC, minimum inhibitory concentration.

bial-resistant $H$. pylori isolates demonstrated improvement of susceptibility as demonstrated by the downward MIC shifting, independent of acid suppression. In particular, for the tegoprazan-treated $H$. pylori isolates, significant susceptibility acquisitions were identified. These susceptibility improvements were most frequently observed in the metronidazole-resistant $H$. pylori isolates. For metronidazole-resistant $H$. pylori isolates, susceptibility acquisitions occurred in $20.6 \%$ of patients and MIC improvement occurred in more than half (52.4\%). Moreover, the rate of susceptibility acquisition in the tegoprazan-treated $H$. pylori strains was significantly higher than that in the vonoprazan-treated $H$. pylori strains ( $20.6 \%$ vs $4.7 \%, \mathrm{p}=0.014$ ).
The present investigators could carefully presume that PCAB-containing treatment might overcome metronidazole resistance-related treatment failure. For instance, sequential therapy (standard dose of PPI and $1 \mathrm{~g}$ amoxicillin twice a day per day for 5 days followed by standard dose of PPI, $500 \mathrm{mg}$ clarithromycin twice a day, and $500 \mathrm{mg}$ metronidazole thrice a day for 5 days) has been suggested to be a practical alternative as the first line regimen instead of PPI-triple therapy. ${ }^{19-21}$ As tegoprazan could overcome metronidazole resistance in the present study, PPI could be replaced by tegoprazan in sequential therapy or bismuthbased quadruple therapy, which contains metronidazole as an antibiotic. Recently, various PCABs are available. 
These PCABs commonly inhibit gastric H,K-ATPase, vonoprazan binds the deeper side of a luminal vestibule and tegoprazan binds with the entrance of pump. This difference in mechanism of action seems likely to be one of the reasons for explaining the difference in susceptibility at metronidazole. In addition, it is necessary to study regarding another mechanism of the metronidazole resistance acquired by overexpression of hefA, which is related with efflux pump. ${ }^{16}$

In this study, a significant inhibitory efficacy of tegoprazan against $H$. pylori was achieved when the concentration reached to $128 \mu \mathrm{g} / \mathrm{mL}$. However, in pharmacokinetics study regarding tegoprazan, the highest concentration of tegoprazan in the bloodstream of healthy subject is only about $1.4 \mu \mathrm{g} / \mathrm{mL}{ }^{5}$ The concentration of $128 \mu \mathrm{g} / \mathrm{mL}$ identified in this experiment does not seem to be easily achieved. However, when considering the range of test concentration up to $64 \mu \mathrm{g} / \mathrm{mL}$ regarding anti-H. pylori efficacy of esomeprazole, ${ }^{22}$ there would be considerable difference of concentrations between in vitro and in vivo. Maybe pharmacologists could suggest these differences in the future.

Unfortunately, the improvement in susceptibility acquired by $\mathrm{PCAB}$ administration did not exhibit a significant correlation with resistance-related genetic mutations. Above all, it is believed that the number of subjects was small to prove our hypothesis. In the future, research including a larger number of subjects is needed. In addition, knowledge of novel mechanism of resistance and research design are required to prove the hypothesis for the resistance mechanism. Meanwhile, the resistance mechanisms of $H$. pylori are often known as target alteration, efflux pump activation, decreased permeability, or plasmidmediated resistance. ${ }^{23}$ As demonstrated in the present study, there is no independent risk factor for susceptibility improvement for all types of mutations for the four antimicrobial agents. A potential cause of this unsatisfactory result might be the insufficient number of study subjects. However, another potential explanation is that the improved susceptibility acquired by PCABs might occur via another mechanism, such as efflux pump activation or permeability alteration, which are not represented by genetic mutation. ${ }^{11,24}$ If knowledge of the currently uninvestigated resistance mechanisms of $H$. pylori is improved, discovering new answers to this problem will be possible. Meanwhile, there was no statistically significant relationship between the A2143 mutations of 23rRNA and susceptibility improvement by PCAB administration for clarithromycinresistant $H$. pylori strains in the present study. Therefore, it is difficult to postulate that PCAB-containing therapy might overcome clarithromycin resistance. Further research is warranted to test alternative treatment strategies such as PCAB-containing quadruple therapy, sequential therapy, or concomitant therapy using the superior efficacy of PCABs.

Because of tegoprazan is a recently developed drug, no direct comparison studies have been found. Since tegoprazan has a different binding site from vonoprazan, it shows differences in half-life, efficacy, and serum gastrin levels. As for the difference in efficacy, various clinical studies including tegoprazan are currently in progress. Unfortunately, the difference of mutations in this study did not show statistical significance. Maybe one of reasons could be small number of samples due to limited research resources. Moreover, in order to prove differences of efficacy of more objectively, objective parameters should be more gathered through the investigation of thorough demographics and underlying resistance mechanism in future study.

In conclusion, $\mathrm{PCAB}$ administration could improve the susceptibility of $H$. pylori to antimicrobial resistance, independent of acid suppression. Additionally, resistance-related gene mutations did not significantly affect susceptibility improvement. Further investigations with PCAB-containing eradication treatments should thus be performed.

\section{CONFLICTS OF INTEREST}

No potential conflict of interest relevant to this article was reported.

\section{ACKNOWLEDGEMENTS}

This work was supported by the Seoul National University Bundang Hospital Research Fund (grant number: 062019-033).

\section{AUTHOR CONTRIBUTIONS}

Study concept and design: N.K., J.W.L. Data acquisition: R.H.N., J.E.Y., J.H.S. Data analysis and interpretation: J.W.L. Drafting of the manuscript; critical revision of the manuscript for important intellectual content: N.K., J.W.L., S.M.L., D.H.L. Statistical analysis: J.W.L. Obtained funding: N.K., J.W.L. Administrative, technical, or material support; study supervision: R.H.N., J.E.Y., J.H.S. Approval of final manuscript: all authors. 


\section{ORCID}

Jung Won Lee

https://orcid.org/0000-0002-7945-1618

Nayoung Kim https://orcid.org/0000-0002-9397-0406

Ryoung Hee Nam https://orcid.org/0000-0002-6515-4540

Jeong Eun Yu https://orcid.org/0000-0003-4079-7360

Joo Hee Son https://orcid.org/0000-0001-9645-897X

Sun Min Lee https://orcid.org/0000-0002-8438-3365

Dong Ho Lee https://orcid.org/0000-0002-6376-410X

\section{REFERENCES}

1. Sugano K. Vonoprazan fumarate, a novel potassium-competitive acid blocker, in the management of gastroesophageal reflux disease: safety and clinical evidence to date. Therap Adv Gastroenterol 2018;11:1756283X17745776.

2. Jung YS, Kim EH, Park CH. Systematic review with metaanalysis: the efficacy of vonoprazan-based triple therapy on Helicobacter pylori eradication. Aliment Pharmacol Ther 2017;46:106-114.

3. Murakami K, Sakurai Y, Shiino M, Funao N, Nishimura A, Asaka M. Vonoprazan, a novel potassium-competitive acid blocker, as a component of first-line and second-line triple therapy for Helicobacter pylori eradication: a phase III, randomised, double-blind study. Gut 2016;65:1439-1446.

4. Scarpignato C, Hunt RH. Editorial: potassium-competitive acid blockers for acid-related diseases-tegoprazan, a new kid on the block. Aliment Pharmacol Ther 2019;50:960-962.

5. Han S, Choi HY, Kim YH, et al. Randomised clinical trial: safety, tolerability, pharmacokinetics, and pharmacodynamics of single and multiple oral doses of tegoprazan (CJ-12420), a novel potassium-competitive acid blocker, in healthy male subjects. Aliment Pharmacol Ther 2019;50:751-759.

6. Lee KJ, Son BK, Kim GH, et al. Randomised phase 3 trial: tegoprazan, a novel potassium-competitive acid blocker, vs. esomeprazole in patients with erosive oesophagitis. Aliment Pharmacol Ther 2019;49:864-872.

7. Lee JW, Kim N, Nam RH, et al. High efficacy of finafloxacin on Helicobacter pylori isolates at $\mathrm{pH} 5.0$ compared with that of other fluoroquinolones. Antimicrob Agents Chemother 2015;59:7629-7636.

8. Lee JW, Kim N, Nam RH, et al. Mutations of Helicobacter pylori associated with fluoroquinolone resistance in Korea. Helicobacter 2011;16:301-310.

9. Hwang TJ, Kim N, Kim HB, et al. Change in antibiotic resistance of Helicobacter pylori strains and the effect of A2143G point mutation of $23 \mathrm{~S}$ rRNA on the eradication of $\mathrm{H}$. pylori in a single center of Korea. J Clin Gastroenterol 2010;44:536-543.

10. Kwon YH, Kim JY, Kim N, et al. Specific mutations of penicillin-binding protein $1 \mathrm{~A}$ in 77 clinically acquired amoxicillinresistant Helicobacter pylori strains in comparison with 77 amoxicillin-susceptible strains. Helicobacter 2017;22:e12437.

11. Lee SM, Kim N, Kwon YH, et al. rdxA, frxA, and efflux pump in metronidazole-resistant Helicobacter pylori: their relation to clinical outcomes. J Gastroenterol Hepatol 2018;33:681-688.

12. Tankovic J, Lascols C, Sculo Q, Petit JC, Soussy CJ. Single and double mutations in gyrA but not in gyrB are associated with low- and high-level fluoroquinolone resistance in Helicobacter pylori. Antimicrob Agents Chemother 2003;47:3942-3944.

13. Lee JW, Kim N, Nam RH, et al. Favorable outcomes of culture-based Helicobacter pylori eradication therapy in a region with high antimicrobial resistance. Helicobacter 2019;24:e12561.

14. Villoria A, Garcia P, Calvet X, Gisbert JP, Vergara M. Metaanalysis: high-dose proton pump inhibitors vs. standard dose in triple therapy for Helicobacter pylori eradication. Aliment Pharmacol Ther 2008;28:868-877.

15. Malfertheiner P, Megraud F, O'Morain CA, et al. Management of Helicobacter pylori infection: the Maastricht V/ Florence Consensus Report. Gut 2017;66:6-30.

16. Gatta L, Vakil N, Ricci C, et al. Effect of proton pump inhibitors and antacid therapy on 13C urea breath tests and stool test for Helicobacter pylori infection. Am J Gastroenterol 2004;99:823-829.

17. Choi HS, Park DI, Hwang SJ, et al. Double-dose, new-generation proton pump inhibitors do not improve Helicobacter pylori eradication rate. Helicobacter 2007;12:638-642.

18. Graham DY, Opekun AR, Hammoud F, et al. Studies regarding the mechanism of false negative urea breath tests with proton pump inhibitors. Am J Gastroenterol 2003;98:1005-1009.

19. Yoon K, Kim N. Eradication rates of 10-day sequential therapy for Helicobacter pylori: results of an 8-year prospective study conducted at a tertiary Korean hospital. Korean J Gastroenterol 2019;73:99-104.

20. Lee JW, Kim N, Kim JM, et al. A comparison between 15day sequential, 10-day sequential and proton pump inhibitor-based triple therapy for Helicobacter pylori infection in Korea. Scand J Gastroenterol 2014;49:917-924.

21. Yoon H, Lee DH, Kim N, et al. Meta-analysis: is sequential therapy superior to standard triple therapy for Helicobacter pylori infection in Asian adults? J Gastroenterol Hepatol 2013;28:1801-1809.

22. Gatta L, Perna F, Figura N, et al. Antimicrobial activity of esomeprazole versus omeprazole against Helicobacter pylori. J Antimicrob Chemother 2003;51:439-442.

23. Francesco VD, Zullo A, Hassan C, Giorgio F, Rosania R, Ierardi E. Mechanisms of Helicobacter pylori antibiotic resistance: an updated appraisal. World J Gastrointest Pathophysiol 2011;2:35-41.

24. Hirata K, Suzuki H, Nishizawa T, et al. Contribution of efflux pumps to clarithromycin resistance in Helicobacter pylori. J Gastroenterol Hepatol 2010;25 Suppl 1:S75-S79. 